\title{
TIREOIDECTOMIA UNILATERAL NO CARCINOMA DIFERENCIADO DA TIREÓIDE DE BAIXO RISCO: UMA CIRURGIA INADEQUADA
}

\author{
UNILATERAL THYROIDECTOMY FOR PATIENTS OF THE LOW-RISK GROUP \\ WITH DIFFERENTIATED THYROID CARCINOMA: \\ AN UNSUITABLE SURGERY
}

\author{
Aluízio Rodrigues, ECBC-RJ ${ }^{1}$ \\ Manuel Domingos da Cruz Gonçalves, TCBC-RJ ${ }^{1}$ \\ Marco Antônio Menezes Ferreira ${ }^{1}$ \\ José Gonçalves Velloso, ACBC-RJ ${ }^{1}$ \\ Orlando Marques Vieira, TCBC-RJ ${ }^{2}$
}

\begin{abstract}
RESUMO: Objetivo: Caracterizar os doentes operados de carcinoma diferenciado da tireóide no Hospital Universitário Clementino Fraga Filho (HUCFF); avaliar a divisão destes doentes em alto e baixo riscos e a validade da cirurgia conservadora nos pacientes com carcinoma diferenciado de baixo risco. Método: Foram analisados 169 pacientes operados no HUCFF por carcinoma diferenciado da tireóide. Cento e cinqüenta e dois (90\%) eram mulheres e $17(10 \%)$ homens. A idade variou entre 13 e 85 anos com mediana de 39 anos. Cento e onze tumores $(65,7 \%)$ eram papilíferos e 58 (34,3\%) foliculares. Resultados: Vinte e um por cento apresentaram metástases linfonodais cervicais quando vistos pela primeira vez e 1,7\% metástases a distância. Houve 75 cirurgias unilaterais (lobectomia subtotal ou total) e 94 bilaterais (tireoidectomia subtotal, lobectomia total + lobectomia subtotal ou tireoidectomia total). 127 casos $(75,2 \%)$ foram considerados pacientes de baixo risco e 42 casos $(24,8 \%)$ de alto risco. O seguimento pós-operatório foi conseguido em 155 pacientes: 116 entre os de baixo risco, com seguimento médio de 8,4 anos (extremos: um ano a 21 anos) e 39 entre os de alto risco (seguimento médio cinco anos, extremos um ano e 17 anos). Foram encontrados 13 recidivas tumorais $(8,3 \%)$ e cinco óbitos pelo tumor $(3,2 \%)$ em toda a série: $10(8,6 \%$ recidivas e nenhum óbito nos pacientes de baixo risco e três recidivas $(7,5 \%)$ e cinco óbitos $(12,5 \%)$ nos de alto risco. Nos pacientes de baixo risco submetidos à tireoidectomia parcial houve 7,5\% de recidivas e nos pacientes com tireoidectomia total a ocorrência de recidivas foi de $10 \%$. Não houve óbitos pelo tumor em nenhum dos grupos. Conclusões: Os pacientes com tumores de baixo risco apresentam evolução mais favorável: número igual de recidivas (relativamente aos tumores de alto risco), porém menos graves, e nenhum faleceu pelo tumor. Nos pacientes de baixo risco a cirurgia unilateral mostrou resultados idênticos aos da bilateral.
\end{abstract}

Descritores: Câncer de tireóide; Grupos de risco; Lobectomia total.

\section{INTRODUÇÃO}

Com a divisão do carcinoma diferenciado da tireóide em tumores de baixo e de alto riscos, estabeleceu-se consenso entre os autores de que o tratamento radical (tireoidectomia total $+\mathrm{I}^{131}$ profilático) é a conduta adequada nos carcinomas de alto risco. Nos de baixo risco, embora todos concordem que a tireoidectomia total não traz vantagem sobre a lobectomia total com istmectomia no que se refere à sobrevida do paciente ${ }^{1-8}$, alguns traba-

1. Professor Adjunto do Departamento de Cirurgia da Faculdade de Medicina da UFRJ.

2. Professor Titular do Departamento de Cirurgia da Faculdade de Medicina da UFRJ. Membro Titular da Academia Nacional de Medicina. Chefe do Serviço de Cirurgia Geral do HUCFF.

Recebido em 2/8/2000

Aceito para publicação em 15/3/2001

Trabalho realizado no Hospital Universitário Clementino Fraga Filho da Universidade Federal do Rio de Janeiro - UFRJ. 
lhos alertam para um maior número de recidivas locais após lobectomia total ${ }^{6,9,10}$. Hay et al. ${ }^{1}$, comparando a incidência de recidiva locais em pacientes submetidos à cirurgia unilateral (lobectomia total) ou bilateral (tireoidectomia subtotal, lobectomia total + lobectomia subtotal ou tireoidectomia total) por carcinoma papilífero da tireóide, encontraram significativa predominância de recidivas com a operação unilateral.

Como este achado não é confirmado em outros estudos 2,3 , decidimos avaliar as recidivas na nossa casuística de 155 casos de carcinoma diferenciado seguidos, usando a mesma divisão em cirurgia uni e bilateral preconizada por Hay et al. ${ }^{1}$. Paralelamente, através da incidência de recidivas e óbitos pelo tumor, procuramos avaliar se os tumores considerados de alto ou de baixo riscos tiveram realmente comportamento biológico diferente.

\section{MÉTODO}

Entre outubro de 1978 e outubro de 1999, 192 pacientes foram operados no Serviço de Cirurgia do Hospital Universitário Clementino Fraga Filho (HUCFF), por carcinoma diferenciado da tireóide. Destes, 23 foram excluídos por terem sido inicialmente operados em outros hospitais ou por serem os dados dos prontuários insuficientes para o estudo.

Os prontuários dos 169 pacientes restantes foram analisados e constituem o material deste trabalho retrospectivo.

Para avaliação de resultados, as operações realizadas foram divididas em dois grupos: operação unilateral (lobectomia total ou subtotal) e operação bilateral (tireoidectomia subtotal clássica, lobectomia total de um lado e subtotal do outro lado e tireoidectomia total).

As recidivas do tumor foram consideradas como: a) locais, quando ocorreram na glândula remanescente ou no leito glandular; b) regionais, quando localizadas nos linfonodos cervicais ou mediastínicos e c) a distância, quando em ossos ou pulmões.

$\mathrm{Na}$ divisão dos doentes em alto e baixo riscos, foi utilizada a classificação por nós estabelecida em 1989 (Tabela 1).
O seguimento pós-operatório foi realizado nos ambulatórios de Cirurgia Geral e no de Endocrinologia de HUCFF. Em alguns pacientes, as informações foram conseguidas por telefone, com seus familiares.

As curvas de sobrevida foram obtidas pelo método de Kaplan-Meier e na comparação destas curvas foi utilizado o teste de Log-Rank; para outras avaliações estatísticas usou-se o teste exato de Fischer, todos realizados pela Comissão de Investigação Científica do Hospital.

\section{RESULTADOS}

Dos 169 casos de carcinoma diferenciado analisados (111 papilíferos - 65,7\% e 58 foliculares - 34,3\%) apenas $17(10 \%)$ eram homens. A idade variou de 13 a 85 anos, com mediana de 39 anos. O tumor estava restrito à glândula em 134 casos (12 eram microcarcinomas), envolvia glândula e linfonodos cervicais em 32 casos; glândula, linfonodos e a distância em um caso e glândula e a distância em dois. Assim, 33 pacientes apresentavam metástases em linfonodos quando vistos pela primeira vez $(20 \%)$ e três metástases a distância $(1,7 \%)$.

Cento e vinte e sete casos $(75,2 \%)$ foram incluídos entre os tumores de baixo risco e os restantes $42(24,8 \%)$ nos de alto risco.

Houve 75 cirurgias unilaterais e 94 bilaterais. A extensão da cirurgia, de acordo com o grupo de risco, pode ser visto na Tabela 2.

Vinte e sete pacientes necessitaram reoperação: a) 10 por cirurgia inicial inadequada para câncer, sendo três em tumores de baixo risco (em um foi encontrado carcinoma residual) e sete entre os de alto risco (em três casos havia microcarcinoma residual); b) seis pacientes por tireoglobulina persistentemente elevada no plasma, todos em tumores de baixo risco e em três deles foi detectado microcarcinoma residual; c) dois, por recidiva de nódulo no lobo remanescente mas o exame histopatológico da peça mostrou, em ambos, lesões benignas; d) nove, por recidiva tumoral clínica, que a patologia confirmou a natureza maligna das recidivas nos nove casos.

O seguimento pós-operatório foi conseguido em 155 pacientes, 116 com tumores considerados de baixo risco

Tabela 1

Carcinoma diferenciado da tireóide

Classificação dos grupos de risco

\begin{tabular}{l|l}
\hline \multicolumn{1}{c|}{ I- Tumor de alto risco } & II- Tumor de baixo risco \\
\hline - Doentes entre 45 e 65 anos, quando houver: & - Doentes com menos de 45 anos com tumor bem diferenciado \\
- Invasão extratireoidiana & e sem metástases a distância. \\
- Tumor $>4 \mathrm{~cm}$ & $\begin{array}{l}\text { Doentes entre } 45 \text { e } 65 \text { anos com tumor bem diferenciado e } \\
\text { - Metástases em linfonodos cervicais }\end{array}$ \\
- Tumor pouco diferenciado & \\
- Presença de metástases a distância & \\
\hline
\end{tabular}


Tabela 2

Cirurgias realizadas -169 casos

\begin{tabular}{|c|c|c|c|c|}
\hline & Baixo risco & Alto risco & Total & Unilateral/bilateral \\
\hline \multicolumn{5}{|l|}{ Na glândula } \\
\hline Lobectomia subtotal & 8 & 1 & 9 & \multirow{2}{*}{$\begin{array}{l}\text { Cirurgia unilateral } \\
\text { (75) }\end{array}$} \\
\hline Lobectomia total & 63 & 3 & 66 & \\
\hline Tireoidectomia subtotal & 17 & 5 & 22 & \multirow{3}{*}{$\begin{array}{l}\text { Cirurgia bilateral } \\
\qquad(94)\end{array}$} \\
\hline Lobectomia total + lobectomia subtotal & 24 & 11 & 35 & \\
\hline Tireoidectomia total & 15 & 22 & 37 & \\
\hline \multicolumn{5}{|l|}{ Em linfonodos } \\
\hline Exérese & 14 & 14 & 28 & \\
\hline Dissecção modificada & 4 & 1 & 5 & \\
\hline Total & 18 & 15 & 33 & \\
\hline
\end{tabular}

(seguimento entre um mês e 21 anos, com média de 8,4 anos) e 39 considerados de alto risco (seguimento entre um mês e 17 anos, com média de cinco anos). Setenta e nove pacientes foram seguidos por mais de cinco anos e 30 por mais de dez anos.

Houve dez recidivas $(8,6 \%)$ e nenhum óbito pelo tumor no grupo de baixo risco e três recidivas $(7,5 \%)$ e cinco óbitos pelo tumor $(12,8 \%)$ no de alto risco. A incidência de recidivas nos dois grupos não mostrou significância estatística $(\mathrm{p}=0,57)$.

Nos pacientes de baixo risco, as recidivas foram locais em cinco, locorregional em um, local + distância (pulmão e osso) em um e regionais em três. Todos foram reoperados e, com exceção do paciente com metástases a distância em tratamento com $\mathrm{I}^{131}$, estão livres de doença entre dois e 18 anos da primeira cirurgia. Cumpre registrar que das sete recidivas locais (cinco isoladas e duas associadas a outras localizações) em cinco tratavam-se apenas de microcarcinomas.

Nos pacientes de alto risco, as recidivas encontradas foram regional em um, regional e a distância em um e a distância em um. Apenas o paciente com recidiva regional (linfonodo cervical) está bem sem sinais da doença sete anos após o início do tratamento; dos outros dois, com metástases a distância, um continua vivo com a doença e o outro foi considerado óbito pelo câncer (paciente com metástases generalizadas quando visto pela última vez e não mais retornou).

As curvas de sobrevida nestes dois grupos, considerados apenas os óbitos pelo câncer, podem ser vistas na Figura 1.

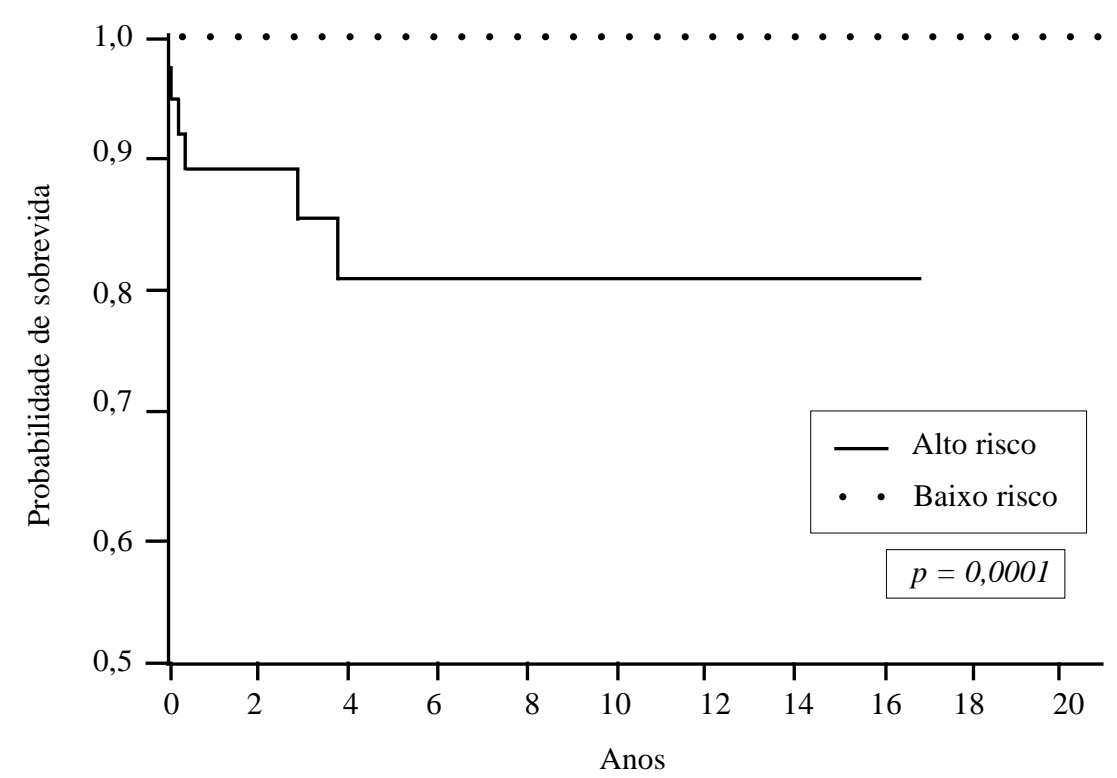

Figura 1 - Probabilidade de sobrevida entre pacientes de alto e baixo risco pelo método de Kaplan-Meier. 
Considerando, agora, as recidivas ocorridas nos pacientes de baixo risco de acordo com a cirurgia a que foram submetidos, encontramos cinco recidivas $(7,5 \%)$ com a cirurgia unilateral (66 casos) e outras cinco ( $10 \%)$ com a bilateral (50 casos). Esta diferença, contudo, não atingiu significância estatística $(\mathrm{p}=0,44)$.

A Figura 1 mostra a incidência cumulativa destas recidivas de acordo com a extensão da cirurgia.

Os resultados obtidos nos 133 pacientes seguidos por mais de um ano, de acordo com o grupo de risco e a extensão da cirurgia, podem ser vistos na Tabela 3 .

\section{DISCUSSÃo}

O câncer da tireóide é talvez o único tumor do organismo capaz de apresentar um largo espectro de comportamento biológico, variando do carcinoma diferenciado, reconhecidamente de baixa agressividade, até o carcinoma anaplásico, um dos tumores de maior letalidade na espécie humana.

Entretanto, cerca de 10 a $20 \%$ dos carcinomas diferenciados podem apresentar comportamento mais agressivo, levando o paciente a recidivas e óbitos. Daí a controvérsia que persiste até hoje sobre a cirurgia mais adequada ao carcinoma diferenciado da tireóide.

Com a definição de fatores de risco capazes de influir no comportamento biológico destes tumores, ocorrida nas duas últimas décadas, foi possível dividir o carcinoma diferenciado em dois grupos: os de baixo risco, de excelente prognóstico, e os de alto risco com altos índices de recidivas e óbitos. Isto permitiu a diversos autores estabelecer tratamento diferenciado para estes grupos: lobectomia total e istmectomia, sem complementação radioterápica para a maioria dos pacientes de baixo risco e tireoidectomia total complementada com I ${ }^{131}$ profilático ou terapêutico ${ }^{11-21,4,2}$ nos de alto risco.

Baseados na fórmula de Hay et al. ${ }^{22}$ e nos trabalhos de Tubiana et al. ${ }^{23}$ Byar et al. ${ }^{24}$ e Harwood et al. ${ }^{25}$, que analisaram os fatores de prognóstico no carcinoma diferenciado da tireóide, estabelecemos uma classificação de grupos de risco, fundamentados naqueles fatores, porém mais prática e objetiva do que as até então existentes e que se mostrou muito semelhante às classificações que se seguiram, descritas por Cady et al. ${ }^{18} \mathrm{e}$ por Kukkonem et al. ${ }^{26}$

Em trabalho anterior já havíamos concluído ser está classificação eficaz na avaliação do comportamento biológico mais ou menos agressivo destes tumores ${ }^{4}$. Os resultados agora obtidos com a análise de 155 pacientes operados e seguidos no HUCFF confirmam os anteriormente encontrados.

Dos 116 pacientes operados e considerados de baixo risco pela classificação adotada, nenhum veio falecer pelo câncer, em seguimento médio de 8,4 anos. Já entre os 39 classificados como de alto risco, cinco faleceram pelo tumor $(12,8 \%)$, em seguimento médio de cinco anos. A probabilidade de sobrevida dos pacientes nestes dois grupos, avaliados pelo método de Kaplan-Meier, mostra curvas significativamente diferentes $(\mathrm{p}<0,001)$ (Figura 1).

Tabela 3

Cirurgias realizadas e resultados - Seguimento $>1$ ano ou até o óbito de acordo com o grupo de risco - 133 casos

\begin{tabular}{|c|c|c|c|c|}
\hline & \multicolumn{2}{|c|}{ Baixo risco (99 casos) } & \multicolumn{2}{|c|}{ Alto risco (34 casos) } \\
\hline & $\begin{array}{l}\text { Cirurgia unilateral } \\
(53 \text { casos })\end{array}$ & $\begin{array}{l}\text { Cirurgia bilateral } \\
(46 \text { casos })\end{array}$ & $\begin{array}{l}\text { Cirurgia unilateral } \\
(3 \text { casos })\end{array}$ & $\begin{array}{l}\text { Cirurgia bilateral } \\
(31 \text { casos })\end{array}$ \\
\hline $1-4$ anos e 11 meses & $\begin{array}{l}\text { Vivo e bem } \\
\text { ( } 21 \text { casos) }\end{array}$ & $\begin{array}{l}\text { Vivo e bem } \\
\text { (11 casos) }\end{array}$ & $\begin{array}{l}- \text { Vivo e bem } \\
\text { ( } 1 \text { caso) } \\
\text { - Óbito pelo câncer: } \\
1 \text { caso }(<1 \text { ano })\end{array}$ & $\begin{array}{l}\text { - Vivo e bem } \\
\text { ( } 12 \text { casos) } \\
\text { - Vivo com metástase: } \\
4 \text { casos } \\
\text { Óbito pelo câncer: } \\
4 \text { casos ( } 3<1 \text { ano e } \\
1 \text { com } 4 \text { anos) }\end{array}$ \\
\hline$>5$ anos & $\begin{array}{l}\text { Vivo e bem } \\
\text { (32 casos) }\end{array}$ & $\begin{array}{l}\text { - Vivo e bem } \\
\text { ( } 32 \text { casos) } \\
\text { - Vivo com metástase } \\
1 \text { caso ( } 16 \text { anos) } \\
\text { - Óbito por outras causas: } \\
2 \text { casos ( } 9 \text { anos) }\end{array}$ & $\begin{array}{l}\text { Vivo e bem } \\
\text { (1 caso) }\end{array}$ & $\begin{array}{l}\text { Vivo e bem } \\
\text { (11 casos) }\end{array}$ \\
\hline Total (1-21 anos) & $\begin{array}{l}\text { Vivo e bem } \\
(53 \text { casos }-100 \%)\end{array}$ & $\begin{array}{l}\text { - Vivo e bem } \\
(43 \text { casos }-93,4 \%) \\
\text { - Vivo com metástase } \\
(1 \text { caso }-2,1 \%) \\
\text { - Óbito por outras causas: } \\
\text { ( } 2 \text { casos }-4,2 \%)\end{array}$ & $\begin{array}{l}\text { - Vivo e bem } \\
(2 \text { casos }-66,6 \%) \\
\text { - Óbito pelo câncer } \\
(1 \text { caso }-33,3 \%)\end{array}$ & $\begin{array}{l}\text { - Vivo e bem } \\
(23 \text { casos - } 74 \%) \\
\text { - Vivo com metástase } \\
\text { (4 casos - 12,9\%) } \\
\text { Óbito pelo câncer } \\
(4 \text { casos }-12,9 \%)\end{array}$ \\
\hline
\end{tabular}


O obituário nulo entre os pacientes de baixo risco na nossa casuística é semelhante ao de outros autores que relatam mortalidade mínima neste grupo de doentes, em casuísticas bem maiores e também com maior tempo médio de seguimento: $1,1 \%$ na experiência de Cady et al. ${ }^{18}, 2 \%$ no de Grant et al. ${ }^{27}$ e $3 \%$ na Shaha et al. ${ }^{28}$. Gemsenjäger et al. ${ }^{13}$ com seguimento médio de sete anos, também não tiveram óbitos em pacientes de baixo risco.

Quanto à incidência de recidivas tumorais, não houve diferença significativa entre os dois grupos, porém foram mais graves nos de alto risco, uma das quais resultou em óbito.

Considerando agora os resultados obtidos com cirurgia uni e bilateral nos pacientes de baixo risco, outro objetivo deste trabalho, podemos concluir que, como não houve óbitos pelo tumor neste grupo de pacientes, a extensão da cirurgia não influiu no obituário, o que está de acordo com a opinião da maioria dos autores 1,2,3,12,14,15,22 .

Vale salientar dois estudos que mostram melhores resultados com a tireoidectomia total no tratamento de pacientes considerados de baixo risco pelos autores. Mazzaferri e Jhiang ${ }^{10}$ analisando 1.134 pacientes com seguimento de até 30 anos encontraram $6 \%$ de mortalidade pelo tumor, quando tratados com tireoidectomia total $+\mathrm{I}^{131}$ pós-operatório, contra $9 \%$ dos que receberam lobectomia total, sem $\mathrm{I}^{131}$. De Groot et al. ${ }^{6}$ estudaram 269 pacientes seguidos por tempo médio de 12 anos que foram divididos em dois grupos: grupo A, pacientes submetidos a operações radicais $+\mathrm{I}^{131}$ pósoperatório, e grupo B, que receberam diversos tipos de cirurgias, radicais e conservadoras, com ou sem $\mathrm{I}^{131}$ pós-operatório. Considerando, em cada grupo, apenas os pacientes incluídos como de "baixo risco", encontraram mortalidade pelo tumor em $0,8 \%$ do grupo A contra $6,1 \%$ do grupo B.

Os resultados destes estudos, contudo, não resistem a uma análise crítica mais cuidadosa. Nos dois trabalhos, o critério usado para classificar os grupos de risco apresenta algumas imperfeições. Nenhum deles leva em consideração a idade dos pacientes e o grau de diferenciação do tumor (bem diferenciado ou pouco diferenciado) como fatores prognósticos para suas classificações. De Groot não inclui também o tamanho do tumor e, no estudo de Mazzeferri, estão incluídos no grupo de pacientes selecionados para a avaliação do tratamento (estádios 2 e 3 da classificação empregada) tumores que variam entre $1,5 \mathrm{~cm}$ a mais de $4,5 \mathrm{~cm}$ de diâmetro. Estes tumores volumosos (> $4 \mathrm{~cm}$ ) em pacientes com mais de 45 anos, são classificados como de alto risco por todos os autores que estudaram o assunto $4,18,23,26,33,34$. Vale ainda salientar que quando De Groot usou como pacientes de baixo risco, apenas aqueles com menos de 45 anos, tumores de $2,5 \mathrm{~cm}$ e intratireoidianos (critério usado por outras classificações para considerar o tumor de baixo risco), não houve morte pelo tumor, quer no grupo submetido à conduta radical quer no grupo com tratamento conservador, embora tenha ocorrido maior número de recidivas neste último, mas sem significância estatística.
Comparando na nossa casuística, a incidência de recidivas após cirurgia unilateral com as ocorridas após cirurgia bilateral no grupo de baixo risco, vimos que ela foi maior na cirurgia bilateral ( $10 \%$ contra $7,5 \%$ na unilateral) embora sem atingir significância estatística, o que mostra que, também sob este aspecto, a cirurgia mais alargada não mostrou melhores resultados, confirmando os achados de $\mathrm{Cady}^{2}$, Shaha ${ }^{3}$ e Balan ${ }^{12}$.

Do nosso conhecimento, os únicos trabalhos que mostram incidência maior de recidivas locais com a cirurgia unilateral em pacientes de baixo risco são os de Grant et al. ${ }^{9}$ e Hay et al. ${ }^{1}$ ambos de Mayo Clinic.

No entanto, eles não separam as recidivas no leito vascular da tireóide (não preveníveis pela tireoidectomia total) das ocorridas em restos tireoidianos, bem como não especificam se tratavam-se de verdadeiras recidivas ou apenas microcarcinomas.

No nosso material, das seis recidivas encontradas em tecido tireoidiano remanescente, cinco eram microcarcinomas. A importância clínica destes microcarcinomas é questionada por vários autores ${ }^{8,16,21,29}$, inclusive por defensores da tireoidectomia total sistemática ${ }^{30,31}$. Shaha ${ }^{20}$ refere de $40 \%$ a $78 \%$ de multicentricidade nos carcinomas papilíferos, relatados na literatura, e a recidiva clínica do tumor em lobo remanescente da tireóide fica entre 6 e $9 \%$ dos casos o que mostra que a imensa maioria dos microfocos carcinomatosos nunca chegou a apresentar manifestações clínicas. Harach ${ }^{32}$, na Finlândia, encontrou tantos microcarcinomas em peças cirúrgicas e em autopsias que considera este achado um fato normal sem significado patológico.

Com relação aos trabalhos de De Groot et al. ${ }^{6}$ e de Mazzaferri et al. ${ }^{10}$, que também referem maior incidência de recidiva com a cirurgia unilateral em pacientes por eles rotulados de baixo risco. Vale lembrar que os critérios por eles usados para caracterizar estes pacientes, como já foi discutido, são diferentes dos empregados por outros autores, o que dificulta a comparação de resultados.

Cumpre ainda salientar que das 10 recidivas locais ou regionais da nossa casuística, em nove, os pacientes estão clinicamente curados, com dois a 18 anos de seguimento, sendo que sete com mais de 10 anos.

A divisão dos pacientes portadores de carcinoma diferenciado da tireóide em alto e baixo riscos mostrou-se mais uma vez válida na previsão do comportamento biológico do tumor, em termos de recidivas ou de óbitos pelo câncer.

Nos pacientes de baixo risco, a cirurgia unilateral apresentou índice de recidivas estatisticamente semelhante ao da bilateral (incidência ligeiramente maior com esta última) e não influiu na sobrevida dos pacientes $(0 \%$ de óbitos pelo tumor).

Estas constatações permitem afirmar que a cirurgia unilateral é adequada ao tratamento do carcinoma diferenciado da tireóide de baixo risco. 


\begin{abstract}
Background: Recent studies challenge the efficacy of unilateral resection in the prevention of recurrences in differentiated thyroid carcinoma of low-risk group. Our objectives were to compare recurrence rates after unilateral and bilateral resections in our so-called low-risk group and to evaluated the accuracy of the criteria used to classify patients into high and low-risk group. Methods: We evaluate outcome of 169 patients who underwent surgery for differentiated thyroid carcinoma at the HUCFF. They were divided in 2 groups: low-risk patients $(n=127)$ and high-risk patients $(n=42)$. Unilateral resection was undertaken in 71 patients of the low-risk group and in 4 of the high-risk group; bilateral resection was the operation used in 56 and 38 patients, respectively, in each of these groups. Results: Follow-up was obtained in 155 patients in a mean period of 8.4 years for 116 low-risk patients and 5 years for 39 high-risk ones. In high-risk group recurrence occurred in 3 patients (7.5\%) and there were 5 tumor related deaths (12.5\%). In the low-risk group, there were 13 recurrences $(8,3 \%)$ but no death occurred. The difference in recurrence rate between groups does not attain statistical e significance $(p=0.57)$. Recurrence occurred in $5(7.5 \%)$ in the low-risk patients submmited to unilateral resection and in 5 (10\%) of those submmited to bilateral resection. Conclusions: The division of patients with differentiated thyroid carcinoma in high and low-risk groups is useful is predicting more aggressive recurrences and tumor-related deaths. Unilateral resection in low-risk patients is not associated with higher recurrence rates.
\end{abstract}

Key Words: Thyroid cancer; Risk-groups; Total lobectomy.

\title{
REFERÊNCIAS
}

1. Hay, I.D.; Grant, C.S.; Bergstralh, E.J. et al: Unilateral total lobectomy: Is it sufficient surgical treatment for patients with AMES low-risk papillary thyroid carcinoma ? Surgery, 124:958-66, 1998.

2. Cady, B.: Our AMES is true: how an old concept still hits the major, risk group assignment points the arrow to rational therapy selection in differentiated thyroid cancer. A, J. Surg, 174:461-8, 1997.

3. Shaha, A.R.; Shah, J.R.; Lorée, T.P.: Low-risk differentiated thyroid cancer: the need for selective treatment. Ann Surg Oncol., 4:328-33, 1997.

4. Rodrigues, R.; Jaccoud, G.; Faciola, P.J. et al: A conduta cirúrgica seletiva no carcinoma diferenciado da tireóide - Ênfase especial aos grupos de risco. Rev. Col. Bras. Cir., 18:182-7, 1991.

5. Rossi, A.L.; Cady, B.; Silverman, M.L. et al: Currents results of conservative surgery for differentiated thyroid carcinoma.World J. Surgery, 10:612-22, 1986.

6. Degroot, L.Y.; Kaplan, E.L.; Straus, F.H. et al: Does the method of management of papillary thyroid carcinoma make a difference in outcome ? World J. Surg., 18:123-30, 1994.

7. Samaan, N.A.; Macheshwari, Y.K.; Nader, S.: Impact of therapy for differentiated carcinoma of the thyroid: an analysis of 706 cases. J. Clin. Endoc. Metabol. 56:1121, 1983.

8. Smith, S.A.; Hay, I.D.; Goellner, J.R. et al: Mortality from papillary thyroid carcinoma. A case control study of 56 lethal cases. Cancer, 62:1381-8, 1988.

9. Grant, C.S.; Hay, I.D.; Gough, I.R. et al: Local recurrence in papillary thyroid carcinoma: Is extent of surgical resection important? Surgery, 104:954-62, 1988.

10. Mazzaferri, E.L. e Yhiang, S.M.: Long-term impact of inicial surgical and medical therapy on papillary and folicular thyroid cancer. Am. J. Medic. 97:418-28, 1994.
11. Andry, G.; Chantrain, G.; Glabbeke, M. et al: Papillary and follicular thyroid carcinoma. Individualization of the treatment according to the prognosis of the disease, Eur. J. Cl. Oncol., 24:1611-16, 1988.

12. Ballan, K.K.; Raduf, A.H.; Critchley, M.: Outcome of 249 patients attendind a nuclear medicine department with well differentiated thyroid cancer: a 23 years review. Brit J. Radiol., 67:283-91, 1994.

13. Gemsenfäger. E.; Heitz, P.U.; Martina, B.: Selective treatment of differentiated thyroid carcinoma. World J. Surg., 21:546-52, 1997.

14. Greebe, S.K.G.; Hay, I.D.: Folicular thyroid cancer. Endoc. Metab. Cl. North Am., 24-761-90, 1995.

15. Smith, S.A.; Hay, I.D.; Goellner, J.R. et al: Mortality from papillary thyroid carcinoma. A case-control study of 56 lethal cases. Cancer, 62:1381-8, 1988.

16. Cady, B.: Arguments against total thyroidectomy. Probl. Gen. Surg., 14:44-51, 1997.

17. Cady, B.; Rossi, R.; Silverman, M. et al: Further evidence of the validity of risk group definition in differentiated thyroid carcinoma. Surgery 98: 1171-8, 1985.

18. Cady, B,; Rossi, R.: An expanded view of risk-group definition in differentiated thyroid carcinoma. Surgery, 104: 947-53, 1988.

19. Shah, J.P.; Loree, T.R.; Darker, D. et al: Lobectomy versus total thyroidectomy for differentiated carcinoma of the thyroid. A matched-pair analysis. Am. J. Surg., 166:331-5, 1993

20. Shaha, A.R.; Jaffe, B.M.: Completion thyroidectomy: a critical appraisal. Surgery. 112:1148-53, 1993.

21. Shah, J.P.; Loree, T.R.; Darker, D. et al: Prognostic factors in differentiated carcinoma of the thyroid gland. Am J. Surg., 164:658-61, 1992.

22. Hay, I.D.; Grant, C.S.; Taylor, W.F. et al: Ipsilateral lobectomy versus bilateral lobar resection in papillary thyroid carcinoma: a retrospective analysis of surgi- 
cal outcome using a novel prognostic scoring system. Surgery, 102:1088-95, 1987 .

23. Tubiana, M.; Schulumberger, M.; Rougier, P. et al: Longterm results and prognostic factors in patients with differentiated thyroid carcinoma. Cancer, 55:794-804, 1985.

24. Byar, D.; Green, S.; Dor, P. et al: A prognostic index for thyroid carcinoma. A study of the EORTC thyroid cancer cooperative group. Eur. J. Cancer, 13-1033, 1985.

25. Harwood, J.; Clark, O.H.; Dumphy, J.E.: Significance of lymph mode metastasis in differentiated thyroid cancer. Am. J. Surg., 136:107-12, 1978.

26. Kukkonem, S.T.; Haapiainen, R.K.; Franssila, K.O. et al: Papillary thyroid carcinoma: the new, age-related TNM classification system in a retrospective analysis of 199 patients. World J. Surg., 14:837-42, 1990

27. Grant, C.S.; Hay, I.D.: Staging and prognosis in differentiated thyroid carcinoma. Probl. Gen. Surg., 14:34-43, 1997.

28. Shaha, A.R.; Loree, T.R.; Shah, J.P.: Prognostic factors and risk group analysis in folicular carcinoma of the thyroid. Surgery, 118:1131-8,1995.

29. Hay, I.D.; Grant, C.S.; Van Heerden, M.B. et al: Papillary thyroid microcarcinoma: a study of 535 cases observed in a 50-year period. Surgery, 112:1139-47, 1992.

30. Samaan, N.A.; Macheshwari, Y.K.; Nader, S.: Impact of therapy for differentiated carcinoma of the thyroid: an analysis of 706 cases. J. Clin. Endoc. Metabol., 56:1131-38, 1983 .

31. De Groot, L.Y.; Kaplan, E.L.; McCormick, M. et al: Natural history, treatment and course of papillary thyroid carcinoma. J. Clin. Endoc. Metabol., 71:414-24, 1990.
32. Harach, H.R.; Franssila, K.O.; Wasenius, V.M.: Occult papillary carcinoma of the thyroid: a "normal" finding in Finland. A systematic autopsy study. Cancer, 56:531-8, 1985.

33. Noguchi, A.; Murakami, N.; Kawamoto, H.: Classification of papillary cancer of the thyroid based on prognosis. World J. Surg., 18:552-8, 1994.

34. Hay, I.D.; Bergstralh, E.J.; Goellner, Y.R. et al: Predicting outcome in papillary thyroid carcinoma: development of a reliable prognostic scoring system in a cohort of 1779 patients surgically treated at ane institution during 1940 through 1989. Surgery, 114:1050-8, 1996.

Endereço para Correspondência

Dr. Aluízio Rodrigues

Rua Smith Vasconcelos, 81

22241-160 - Rio de Janeiro-RJ 